\title{
Optimizing Height and Packing Density of Oriented One- Dimensional Photocatalysts for Efficient Water Photoelectrolysis
} Huajun Zhou, ${ }^{\dagger,}$ Huixu Deng, ${ }^{\S}$ Seyed A. Ghetmiri," Husam H. Abu-Safe," Shui Q. Yu," Xiaodong Yang, ${ }^{*}$, and Z. Ryan Tian*,t,

\footnotetext{
${ }^{\dagger}$ Department of Chemistry and Biochemistry, ${ }^{\dagger}$ Institute of Nanoscale Materials Sciences and Engineering, and ${ }^{\|}$Department of Electrical Engineering, University of Arkansas, Fayetteville, Arkansas 72701, United States

${ }^{\S}$ Department of Mechanical and Aerospace Engineering, Missouri University of Science and Technology, Rolla, Missouri 65409, United States
}

Supporting Information

ABSTRACT: Dependences of water-photoelectrolysis efficiency on heights and packing densities of vertically arrayed $\mathrm{ZnO}$ nanorods (NRs) and $\mathrm{ZnO}$ microrods (MRs) were systematically studied for the first time over a wide range of light incidence angles, under the direction of nanooptics simulation. In the photoelectrolysis, dense NRs of $1.8 \mu \mathrm{m}$ in height afforded the highest photocatalytic efficiencies, and further increases of the height kept lowering down the photocatalytic efficiencies, while sparse MRs taller in height consistently afforded better electrolyte penetration and higher photocurrent densities especially at higher angles of incident light. The experimental results are in line with the nanooptics simulation. This new finding is generally applicable to advancing solar-energy conversions, optics, and optoelectronics using oriented one-dimensional micro/nanocrystallites.

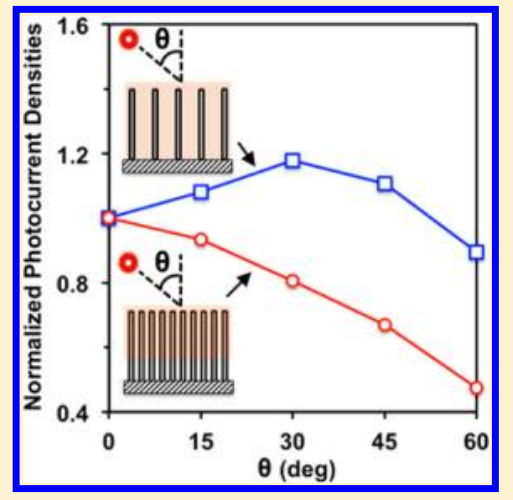

\section{INTRODUCTION}

Oriented arrays of semiconducting microrods (MRs) and nanorods (NRs) are among the most promising photoelectrodes for water photoelectrolysis in a photoelectrochemical (PEC) cell, in comparison with planar films of the same semiconductors. ${ }^{1-3}$ This is because the oriented arrays provide not only a direct path for photogenerated charge carriers to be more efficiently collected, ${ }^{4}$ but also a short transport distance ${ }^{5}$ (i.e., the radius of a rod) for minority charge carriers to reach the rod's surface for performing the redox reactions. Therefore, enormous efforts have been put on maximizing the solar energy conversion efficiencies over a broad range of wavelengths and light incidence angles using the MRs and NRs. To improve the light absorption over wide angles of incidence, coatings of photovoltaic materials ${ }^{6}$ and antireflection layers ${ }^{7}$ have been employed.

Herein we demonstrate that, without the help of abovementioned coatings, semiconductor nano- and microrods in vertically oriented arrays with optimized heights and packing densities can afford optimal water photoelectrolysis over wide angles of incident light. To mimic the field photocatalysis under natural sunlight, the PEC cell setup employed simulated sunlight whose beam spot covered each photoanode's entire surface (Scheme 1), which is different from those in literature with the beam diameters smaller than the photoelectrodes. ${ }^{3,8}$ The angles of incidence $(\theta)$ are defined as the angles between the light and the normal direction of rods' top surfaces and equivalent to the angle shown in the Scheme 1. For the first
Scheme 1. Schematic Representation of the Setup of Photoelectrochemical (PEC) Cell: WE, ZnO Working Electrode; RE, Ag/AgCl Reference Electrode; CE, Counter Electrode; $\theta$, Angle of Incident Light

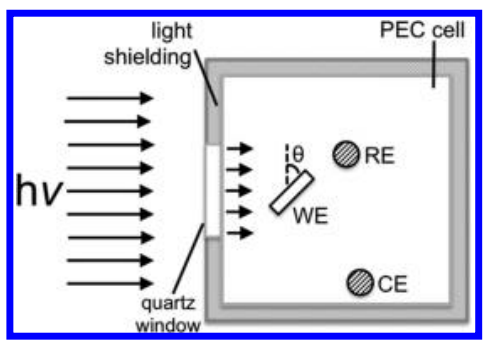

time, our results disclosed that the surface areas, absorption volumes, and packing densities need to be balanced in order for the arrayed photocatalytic NRs and MRs to achieve efficient photocatalysis over wide angles of incident light, which may shed new light on conversions of solar energy using photoactive micro/nanomaterials in general.

\section{EXPERIMENTAL METHODS}

Syntheses of Densely Arrayed ZnO-NRs of Different Heights. Densely arrayed ZnO-NRs of different heights were

Received: July 23, 2013

Revised: September 1, 2013

Published: September 6, 2013 
grown sequentially on a same substrate. First, $\mathrm{ZnO}$ nanoseeds were deposited onto an F-doped $\mathrm{SnO}_{2}($ FTO $)$ glass $(1.8 \times 1.0$ $\mathrm{cm}$ ) substrate by two cycles of deposition and decomposition of zinc acetate. ${ }^{9}$ Thereafter, the substrate was immersed into an aqueous solution containing $25 \mathrm{mM} \mathrm{Zn}\left(\mathrm{NO}_{3}\right)_{2}$ and $25 \mathrm{mM}$ hexamethylenetetramine (HMTA) at $92{ }^{\circ} \mathrm{C}$ for $2.5 \mathrm{~h}$. Then the substrate was washed clean with double deionized (DDI) water, annealed at $400{ }^{\circ} \mathrm{C}$ in air for $0.5 \mathrm{~h}$, and subject to a PEC measurement. The substrate was then washed clean using DDI water and immersed into a fresh solution containing $25 \mathrm{mM}$ $\mathrm{Zn}\left(\mathrm{NO}_{3}\right)_{2}, 25 \mathrm{mM} \mathrm{HMTA}$, and $6 \mathrm{mM}$ polyethylenimine (PEI) at $92{ }^{\circ} \mathrm{C}$ for $2.5 \mathrm{~h}$ for a second crystal growth. Thereafter, the substrate was likewise cleaned, annealed, and subject to a second PEC measurement under the same experimental conditions and settings. Then the substrate was subject to the third, fourth, and fifth growths and measurements. These experiments were repeated on four substrates to obtain an average.

Syntheses of Sparsely Arrayed ZnO-MRs of Different Heights. Sparsely arrayed $\mathrm{ZnO}$-MRs of different heights were grown sequentially on the same substrate following a modified procedure. ${ }^{10}$ An FTO glass $(2.4 \times 1.0)$ substrate was ultrasonicated consecutively in acetone, ethanol, isopropyl alcohol, and DDI water each for $10 \mathrm{~min}$, then blown dry with $\mathrm{N}_{2}$ gas, and heated at $200{ }^{\circ} \mathrm{C}$ for $5 \mathrm{~min}$ for further dryness. Then a 50-nm-thick layer of $\mathrm{Au}$ was deposited onto the substrate by a SC7620 sputter coater (Quorum Technologies) and the substrate was annealed at $300{ }^{\circ} \mathrm{C}$ for $1 \mathrm{~h}$. Thereafter, the substrate was immersed into an aqueous solution containing $2.0 \mathrm{mM} \mathrm{Zn}\left(\mathrm{NO}_{3}\right)_{2}$ and $2.0 \mathrm{mM} \mathrm{HMTA}$ at $70{ }^{\circ} \mathrm{C}$ for $6 \mathrm{~h}$. Then the substrate was washed clean with DDI water, annealed at $400{ }^{\circ} \mathrm{C}$ in air for $0.5 \mathrm{~h}$, and subject to a PEC measurement. The substrate was then washed clean using DDI water and immersed into a fresh solution containing $25 \mathrm{mM}$ $\mathrm{Zn}\left(\mathrm{NO}_{3}\right)_{2}, 25 \mathrm{mM}$ HMTA, and $6 \mathrm{mM}$ PEI at $92^{\circ} \mathrm{C}$ for $1 \mathrm{~h}$ for a second crystal growth. Thereafter, the substrate was cleaned likewise, annealed, and subject to a second PEC measurement under the same experimental conditions and settings. Then the substrate was subject to third and fourth growths and measurements. These experiments were repeated on four substrates to obtain the average.

Characterizations. The phase purity and spatial organization of $\mathrm{ZnO} \mathrm{NRs}$ and MRs were characterized by a Rigaku MiniFlex II Desktop X-ray diffractometer using monochromatized $\mathrm{Cu} \mathrm{K} \alpha$ radiation $(\lambda=1.5418 \AA)$ at $30 \mathrm{kV}$ and $15 \mathrm{~mA}$. A continuous scan mode was used to collect the diffraction data from 30 to $60^{\circ}$ at a speed of $0.2^{\circ} / \mathrm{min}$. The morphologies of arrayed rods were investigated under a scanning electron microscope (SEM, e.g., Tescan VEGA II SBH or Philips ESEM XL30 equipped with a field emission gun) operated at $10 \mathrm{kV}$. To minimize charging problems, samples for SEM were coated with thin Au layers. For PL measurements, the samples were excited by a pulsed Nd:Yag laser with the wavelength at $355 \mathrm{~nm}$ and the PL spectra were collected by iHR550 Horiba Jobin Yvon spectrometer at room temperature.

PEC Measurements. The setup of a PEC cell includes a $150 \mathrm{~mL}$ glass bottle with a fused quartz window of $2.0 \mathrm{~cm}$ in diameter. The cell contains an electrolyte solution of $50 \mathrm{~mL} 0.5$ $\mathrm{M} \mathrm{Na}_{2} \mathrm{SO}_{4}$ in double-deionized (DDI) water ( $\mathrm{pH} \sim 5.7$ ), a substrate with arrayed MRs or NRs as the photoanode, a Pt counter electrode $\left(18 \times 5 \times 1 \mathrm{~mm}^{3}\right)$, a KCl-saturated $\mathrm{Ag} / \mathrm{AgCl}$ reference electrode, and a Solar Simulator (ABET Technologies, LS-150-Xe) as the light source. The $150 \mathrm{~W}$ xenon lamp was coupled with an AM 1.5G filter (Newport) to simulate the sunlight source, the incident light intensity was set up to 33 $\mathrm{mW} / \mathrm{cm}^{2}$ (i.e., $33 \%$ of $\mathrm{AM} 1.5 \mathrm{G}$ ) using with a calibrated $\mathrm{Si}$ photodiode, and the beam size was about $5.7 \mathrm{~cm}$. Before the measurement, the electrolyte solution was purged with $\mathrm{N}_{2}$ for $15 \mathrm{~min}$ and then the cell was sealed with parafilm to minimize the interference of $\mathrm{O}_{2}$ from air. The linear sweep voltammagrams were obtained by scanning the bias potential (vs $\mathrm{Ag}$ / $\mathrm{AgCl}$ ) from -0.6 to $1.0 \mathrm{~V}$ for densely arrayed $\mathrm{ZnO}-\mathrm{NRs}$ and from -0.6 to $0.4 \mathrm{~V}$ for sparsely arrayed $\mathrm{ZnO}-\mathrm{MRs}$ at the rate of $25 \mathrm{mV} / \mathrm{s}$ consistently under dark, full-spectrum, and visible/ infrared light illuminations. The scanning voltage window in the latter was narrower in order not to destroy the Au films. During the PEC measurements under full-spectrum light illumination, linear sweep voltammagrams of the photoanodes were recorded with $\theta$ being tuned from $0^{\circ}$ to $60^{\circ}$ with $15^{\circ}$ interval. Dark currents were deducted from currents under fullspectrum light illumination and the resultant photocurrents were normalized by substrate areas to calculate the photocurrent densities. Visible/infrared light illumination was obtained when an additional filter (Andover Corporation, GG420) was used to cover the fused quartz window to block UV light during the full-spectrum light illumination.

\section{RESULTS/DISCUSSION}

The average heights of densely arrayed NRs from one- to fivegrowths were $\sim 0.9$ (Figure 1a), $\sim 1.8$ (Figure 1b), $\sim 2.7$ (Figure 1c), 3.6 (Figure S1a), and $\sim 4.5 \mu \mathrm{m}$ (Figure S1b), respectively, and their corresponding average diameters were $\sim 70,80,90,100$, and $110 \mathrm{~nm}$, respectively. Compared to significant increases in height, the slight increases in diameter proved that the cationic PEI successfully hindered the lateral

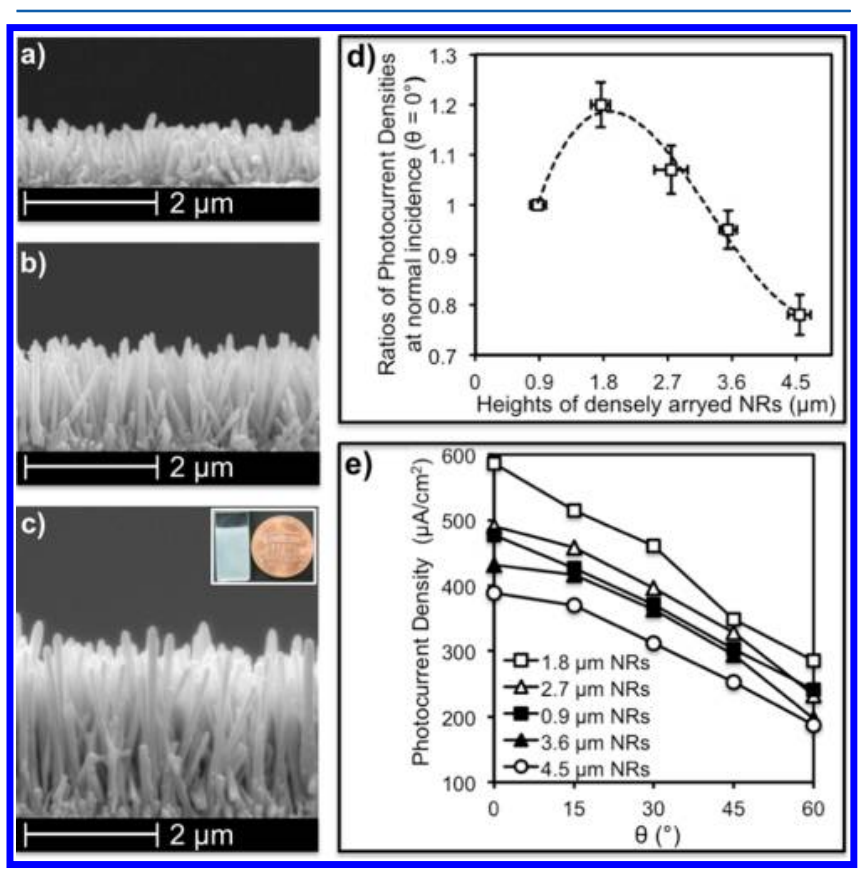

Figure 1. $(\mathrm{a}-\mathrm{c})$ Cross-sectional SEM images of densely arrayed $\mathrm{ZnO}$ NRs of $\sim 0.9$ (a), $\sim 1.8$ (b), and $\sim 2.7 \mu \mathrm{m}$ (c) in height. The inset in (c) is the corresponding optical micrograph of the photoanode. (d) Ratios of photocurrent densities of arrayed NRs of different heights at $V_{\text {bias }}=$ $1.0 \mathrm{~V}$ to that of the $\sim 0.9 \mu \mathrm{m}$ tall NRs at normal incidence $\left(\theta=0^{\circ}\right)$. (e) Correlation between the photocurrent densities of all arrayed NRs at $V_{\text {bias }}=1.0 \mathrm{~V}$ and angles of incidence. 
growth of NRs. ${ }^{4}$ The inset in Figure 1c is a photograph of a 1.8 $\times 1.0 \mathrm{~cm}$ FTO glass substrate with the corresponding arrayed NRs, depicting the uniformity of the NRs across the whole substrate area (the bare top was for clamping the alligator electrode). The dominant (002) peaks in the powder X-ray diffraction (PXRD) patterns of all arrayed NRs (Figure S2a) confirm that all vertical NRs were well aligned along the [001] direction. The peak positions of the photoluminescence (PL) spectra of all arrayed NRs are almost the same (Figure S2b), suggesting their similar surface states and band gaps.

Photocurrents at $V_{\text {bias }}=1.0 \mathrm{~V}$ from visible light (Figure S3) were negligible for all arrayed NRs, proving negligible changes in band gaps due to limited nitrogen-doping from HMTA and PEI during the repeated growths. The onset potentials of photocurrents of all NRs were close to each other (ranging from -0.4 to $-0.3 \mathrm{~V}$ ). Reproducibly, as the heights of NRs increased from $\sim 0.9$ to $\sim 4.5 \mu \mathrm{m}$, the photocurrent densities $\left(V_{\text {bias }}>-0.20 \mathrm{~V}\right)$ of arrayed NRs at normal incidence $\left(\theta=0^{\circ}\right)$ reached maximum for $\sim 1.8 \mu \mathrm{m}$ tall $\mathrm{NRs}$ and then kept decreasing (Figure S4). On this basis, the photocurrent densities of all arrayed NRs at $V_{\text {bias }}=1.0 \mathrm{~V}$ were compared with that of the $\sim 0.9 \mu \mathrm{m}$ tall NRs and the ratios were averaged for four samples (Figure 1d). Importantly, the increased surface areas (Figure S5a) did not necessarily improve photocatalytic efficiencies, which differs from the intuition in surface area dominated heterogeneous photocatalysis. Unanimously, the photocurrent densities of all arrayed NRs decreased as $\theta$ increased from 0 to $60^{\circ}$ (Figure 1e), which reflects the correlation between photocatalytic efficiencies and the light beam's cross-section areas on tilted substrates.

To understand the height- and angle-of-incidence-dependent PEC performances of arrayed NRs, 3D (vector) rigorous coupled wave analysis (RCWA $)^{11}$ was conducted on the optical absorption and optical mode distributions over the densely arrayed NRs. Figure $2 \mathrm{a}$ shows the distributions of the normalized electric field intensity $|E|^{2}$ and the absorption power density $P\left(P=(\omega / 2) \operatorname{Im}[\varepsilon(\omega)] \varepsilon_{0}|E|^{2}\right)^{12}$ for a $0.9 \mu \mathrm{m}$ tall $\mathrm{NR}$ at $\lambda=300 \mathrm{~nm}$ and $\theta=0^{\circ}$. Because the NRs are densely packed, most of the incident light can be absorbed at the top region up to several hundreds of nanometers for each NR (Figure 2a). For this reason, as the heights of NRs increase from 0.9 to $4.5 \mu \mathrm{m}$, the integrated absorption over the wavelength range of $200-400 \mathrm{~nm}$ slightly increase from $90.4 \%$ for $0.9 \mu \mathrm{m}$ tall NRs to $93.8 \%$ for $1.8 \mu \mathrm{m}$ tall NRs and then increase even more slowly (Figure S5b) thereafter. On this basis, the ratios of simulated absorption of all arrayed NRs to that of $0.9 \mu \mathrm{m}$ tall NRs were plotted against the heights (Figure $2 \mathrm{~b}$ ), and the trend is clearly different from that of photocurrent densities. Thus, neither surface areas nor light absorption accounted for the trend in Figure 1d. Because surface states of all samples were preunified from the same annealing process before PEC measurements, no significant band gap shifts were observed, and little to no grain boundary would form from the repeatedly epitaxial growths, ${ }^{13}$ implying that other factors governed the trend in Figure 1d.

Intuitively, charge-transport properties might play different roles for arrayed NRs of different heights. However, Law et al. reported that the electron transport in electrolyte-bathed $\sim 5.0$ $\mu \mathrm{m}$ tall $\mathrm{ZnO}-\mathrm{NR}$ arrays was fast, ${ }^{4}$ indicating charge transport should not be an issue. In literature, photocurrent densities in dye-sensitized solar cells (DSSCs) built of arrayed $\mathrm{ZnO}-\mathrm{NRs}$ were reported to repeatedly increase as the heights increased up to $18-24 \mu \mathrm{m},{ }^{4,13}$ which is different from the trend in Figure 1d.

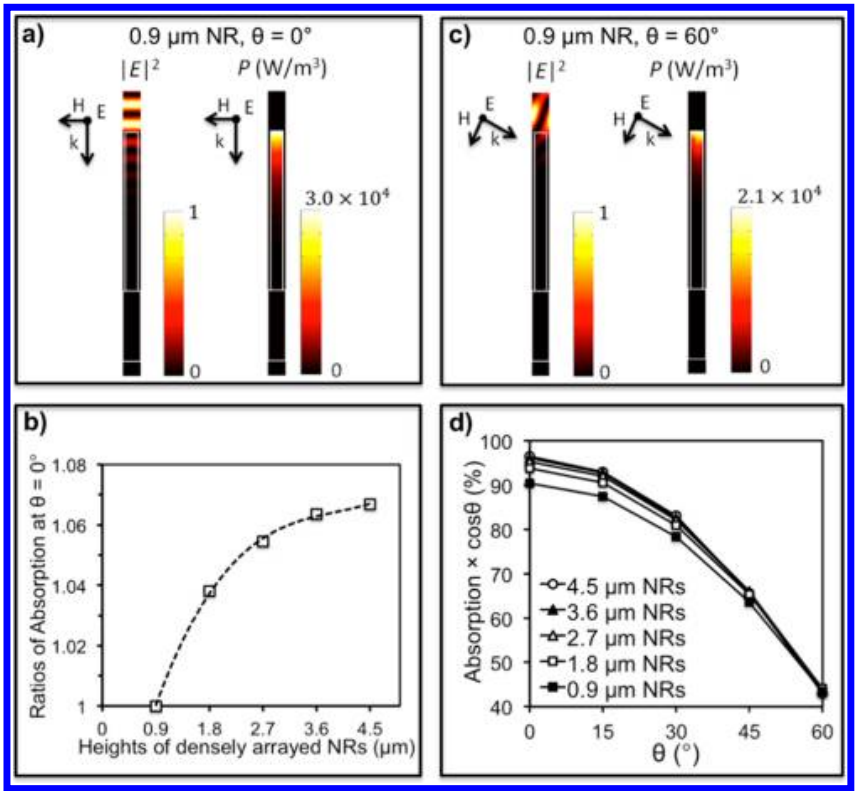

Figure 2. Optics simulation for densely arrayed NRs. (a, c) The distributions of the normalized electric field intensity $|E|^{2}$ and the absorption power density $P$ for $0.9 \mu \mathrm{m}$ tall NR at $\lambda=300 \mathrm{~nm}$ and $\theta=$ $0^{\circ}$ (a) and $\theta=60^{\circ}$ (c). (b) Ratios of simulated absorption integrated over $\lambda=200-400 \mathrm{~nm}$ at $\theta=0^{\circ}$ of all arrayed NRs to that of the 0.9 $\mu \mathrm{m}$ tall NRs. (d) Correlation between simulated (absorption $\times \cos \theta$ ) of all arrayed NRs and $\theta$.

The difference could be due to different electrolyte penetration kinetics that were associated with inter-NRs spacing and pretreatments before measurements. Kim et al. showed that it took $1 \mathrm{~h}$ for an aqueous electrolyte to completely fill the $\mathrm{TiO}_{2}$ nanotubes $(\sim 100 \mathrm{~nm}$ in inner diameter and $\sim 1.8 \mu \mathrm{m}$ in depth) under the illumination of $350 \mathrm{~nm}$ light with the intensity being $2.84 \mathrm{~mW} / \mathrm{cm}^{2} .{ }^{14}$ The electrolyte penetration in the densely arrayed NRs should also be slow because the inter-NRs spacing among most NRs in $\sim 0.9 \mu \mathrm{m}$ tall NRs was about $40-50 \mathrm{~nm}$ (Figure S6) though the stronger light intensities here should help enhance electrolyte penetration. ${ }^{14}$ This was evidenced by the fact that photocurrent densities always became stable during and after the second scan (Figure S7) with each scan taking $64 \mathrm{~s}$. As the heights of NRs increased from $\sim 0.9 \mu \mathrm{m}$ to $\sim 1.8 \mu \mathrm{m}$, the surface areas increased (more than $100 \%$, Figure S5a) and so did the light absorption ( $3.4 \%$, Figure S5b), resulting in an increase in photocurrent densities. Hence, the electrolyte-penetration kinetics in the solutions might only change slightly due to slightly increased NR diameters and thus slightly decreased inter-NRs spacing $(30-40 \mathrm{~nm})$. However, as the heights continued to increase to $\sim 2.7,3.6$, and $4.5 \mu \mathrm{m}$, the light absorption increased in slower rates (Figure S5b), while the inter-NRs spacing among most NRs were $20-30 \mathrm{~nm}, 10-$ $20 \mathrm{~nm}$, and then a few $\mathrm{nm}$, respectively, resulting in much slower electrolyte penetration and much decreased electrolyteaccessible surface areas for redox reactions and, thus, decreased photocurrent densities. As the heights of NRs kept increasing from $\sim 4.5$ to $\sim 13 \mu \mathrm{m}$, more NRs tended to merge and, thus, surface areas kept decreasing, ${ }^{15}$ while electrolyte penetration became slower and slower, which led to continually decreased photocurrent densities.

Figure $2 c$ shows the distributions of the normalized electric field intensity and the absorption power density for a $0.9 \mu \mathrm{m}$ tall NR at $\lambda=300 \mathrm{~nm}$ and $\theta=60^{\circ}$. The optical absorption at $\theta$ 
$=60^{\circ}$ was found to be slightly smaller than that at $\theta=0^{\circ}$, due to the fact that the optical reflection at the interface between the NR and the solution slightly increased thus the absorption decreased. On this basis, the light absorption of densely arrayed NRs of 0.9 to $4.5 \mu \mathrm{m}$ in height at $\theta=0^{\circ}, 15^{\circ}, 30^{\circ}, 45^{\circ}$, and $60^{\circ}$ were integrated over $\lambda=200-400 \mathrm{~nm}$. For each NR array, as $\theta$ increases from $0^{\circ}$ to $15^{\circ}$ and $30^{\circ}$, the optical absorption are almost the same but then slightly decrease as $\theta$ reaches $45^{\circ}$ and $60^{\circ}$ (Figure S8). Because in our experimental setup the projected photon flux upon electrode substrates was proportional to $\cos \theta$, on this basis (absorption $\times \cos \theta$ ) of all arrayed NRs was plotted against $\theta$ (Figure $2 \mathrm{~d}$ ). We found that the (absorption $\times \cos \theta$ ) of all NRs keeps decreasing as $\theta$ increases, which matches the trends of photocurrent densities of all arrayed NRs as a function of $\theta$ (Figure 1e).

To improve the electrolyte penetration, we prepared sparsely arrayed MRs of different heights by modifying a published procedure, ${ }^{10}$ and investigated their height- and angle-ofincidence-dependent PEC performances. Each FTO glass substrate was coated with a $50 \mathrm{~nm}$ thick $\mathrm{Au}$ (111) film to help grow the vertically aligned MRs, ${ }^{16}$ which was confirmed by the dominant (002) peaks in the PXRD patterns (Figure S9a). The heights and diameters of MRs from one to four growths are $\sim 1.6 \mu \mathrm{m} / 0.35 \mu \mathrm{m}$ (Figure 3a), $\sim 2.6 \mu \mathrm{m} / 0.45 \mu \mathrm{m}$ (Figure

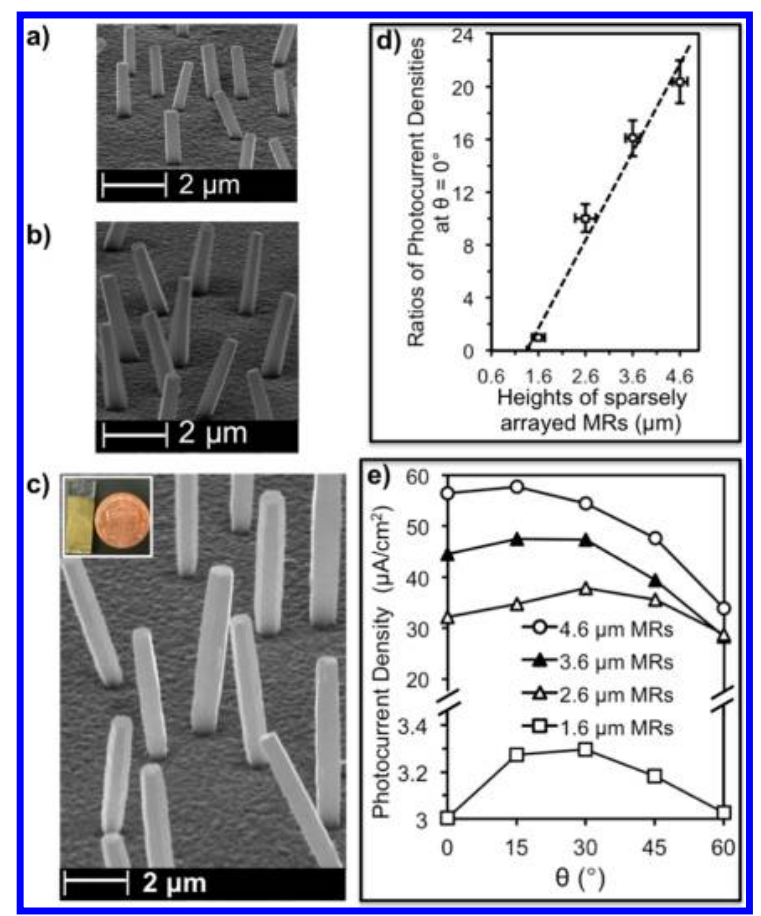

Figure 3. $(\mathrm{a}-\mathrm{c})$ View of SEM images $\left(30^{\circ}\right.$ tilt $)$ of sparsely arrayed ZnO-MRs of $\sim 1.6$ (a), $\sim 2.6$ (b), and $\sim 4.6 \mu \mathrm{m}$ (c) in height obtained after 1st, 2nd, and 4th growths, respectively. The inset in (c) is the optical photograph of the photoanode. (d) Ratios of photocurrent densities at $V_{\text {bias }}=0.4 \mathrm{~V}$ of all arrayed MRs at normal incidence $(\theta=$ $0^{\circ}$ ) to that of $\sim 1.6 \mu \mathrm{m}$ tall MR array. (e) Correlation between photocurrent densities of all arrayed MRs at $V_{\text {bias }}=0.4 \mathrm{~V}$ and $\theta$.

3b), $\sim 3.6 \mu \mathrm{m} / 0.60 \mu \mathrm{m}$ (Figure S10), and $\sim 4.6 \mu \mathrm{m} / 0.75 \mu \mathrm{m}$ (Figure $3 \mathrm{~d})$, respectively. The average spacing $(\sim 1.8 \mu \mathrm{m})$ and packing densities of all arrayed MRs are the same and the heights of MRs in each array are quite uniform, indicating the sequential growths occur on MRs rather than at new spots on the substrates. The heights of MRs increased $\sim 1.0 \mu \mathrm{m}$ after each growth while their diameters slightly increased $(\sim 0.1 \mu \mathrm{m})$, indicating PEI effectively suppressed the lateral growths of MRs. The peak positions of their PL spectra are very similar (Figure S9b), suggesting their similar surface states and band gaps.

The potential windows of the linear sweep voltammagrams of the Au-coated FTO glasses with sparsely arrayed MRs are from -0.6 to $0.4 \mathrm{~V}$, which are narrower than those of FTO glasses with densely arrayed NRs due to the presence of $\mathrm{Au}$ film. For all arrayed MRs, the dark currents and currents generated under visible-light illumination at $V_{\text {bias }}=0-0.4 \mathrm{~V}$ were almost identical (Figure S11), indicating the photocurrents contributing from visible light were negligible. This result further suggests that the band gap shifts from possible nitrogen doping from HMTA and PEI during the repeated growths were negligible, and so was the possible photocatalytic activities from $\mathrm{Au}$ films via surface plasmon resonance (SPR) ${ }^{17}$ in water photoelectrolysis under visible-light illumination. The dark current densities of all arrayed MRs at $V_{\text {bias }}=0-0.4 \mathrm{~V}$ were almost identical and deducted from the current densities obtained under full-spectrum light illumination (Figure S12) and the resultant photocurrent densities of all arrayed MRs at $V_{\text {bias }}=0.4 \mathrm{~V}$ were compared to those of $\sim 1.6 \mu \mathrm{m}$ tall MRs and the ratios from four substrates were averaged. We found that as the heights increased, the photocurrent densities of arrayed MRs keep increasing in a much more drastic fashion and their ratios are 1.0:10.0:16.1:20.4 (Figure 3d). This trend is different from that for densely arrayed NRs, which is interesting when considering their similar surface area increases as a function of height increase (Figure S13a). More interestingly, the photocurrent densities did not necessarily decrease as the angles of incidence increased (Figure 3e). The angles of incidence at which arrayed MRs afforded the highest photocurrent densities were $30^{\circ}$ for arrayed MRs of $\sim 1.6$ and $\sim 2.6 \mu \mathrm{m}$ in height and $15^{\circ}$ for arrayed MRs of $\sim 3.6$ and $\sim 4.6 \mu \mathrm{m}$ in height, indicating the best angles of incidence for trapping light as a function of the heights.

To understand the height- and angles-of-incidence-dependent PEC performances of sparsely arrayed MRs, we performed optics simulations of the light absorption for them. Figure 4a shows the distributions of the normalized electric field intensity and the absorption power density for a $1.6 \mu \mathrm{m}$ tall $\mathrm{MR}$ at $\lambda=$ $300 \mathrm{~nm}$ and $\theta=0^{\circ}$. A large portion of the incident light tends to transmit through the MR-array due to the large inter-MRs spacing, and light absorption is taken place within the entire arrayed MRs. Then the light absorption for all arrayed MRs was integrated over the wavelength range of $200-400 \mathrm{~nm}$, and on that basis we found light absorption for arrayed MRs of 1.6, 2.6, 3.6 , and $4.6 \mu \mathrm{m}$ are $21.5,30.1,39.1$, and $46.3 \%$, respectively (Figure S13b), and the ratios of light absorption are 1:1.40:1.82:2.15 (Figure 4b). Thus, as the heights of sparsely arrayed MRs increased, both light absorption and surface areas almost linearly increased while the electrolyte penetrations remained rapid due to the large inter-MRs spacing $(\sim 1.8 \mu \mathrm{m})$, resulting in consistent increases of the photocurrent densities (Figure 3d).

Figure $4 \mathrm{c}$ shows the distributions of the normalized electric field intensity and the absorption power density for a $1.6 \mu \mathrm{m}$ tall MR at $\lambda=300 \mathrm{~nm}$ and $\theta=60^{\circ}$. The optical absorption at $\theta$ $=60^{\circ}$ was found to be larger than that at $\theta=0^{\circ}$, due to increased photon scattering cross-section areas. On this basis, the light absorption of arrayed MRs of 1.6 to $4.6 \mu \mathrm{m}$ in height were integrated over the wavelengths of $200-400 \mathrm{~nm}$ at angles- 


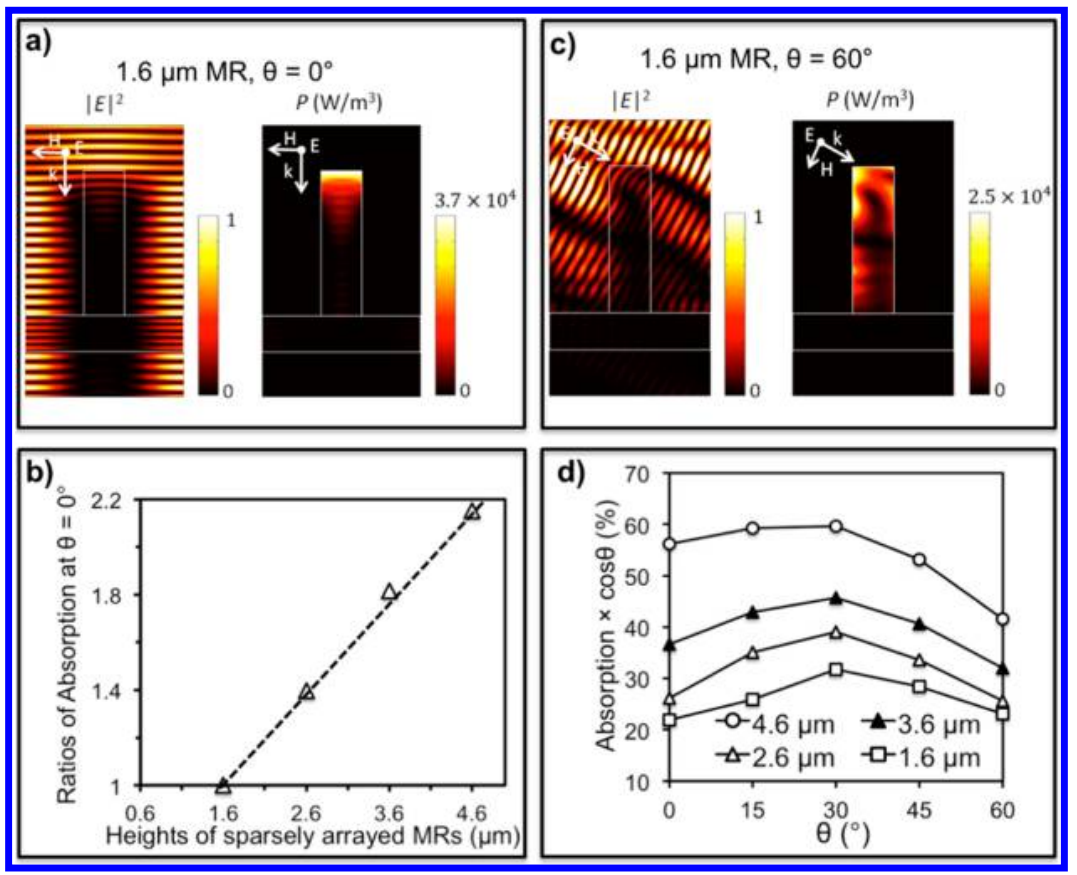

Figure 4. Optics simulation for sparsely arrayed MRs. (a, c) The distributions of the normalized electric field intensity $|E|^{2}$ and the absorption power density $P$ for a $\sim 1.6 \mu \mathrm{m}$ tall MR at $\lambda=300 \mathrm{~nm}$ at $\theta=0^{\circ}$ (a) and $\theta=60^{\circ}$ (c). (b) Ratios of simulated absorption at normal incidence $\left(\theta=0^{\circ}\right)$ of all arrayed MRs to that of $\sim 1.6 \mu \mathrm{m}$ tall MR array. (d) Correlation between simulated (absorption $\times \cos \theta$ ) of all arrayed MRs and $\theta$.

of-incidence from $0^{\circ}$ to $60^{\circ}$. We found that as $\theta$ increases, the light absorption unanimously increases for all arrayed MRs (Figure S14). After the absorption was normalized by the incoming light flux, the normalized absorption (i.e., absorption $\times \cos \theta)$ of all arrayed MRs was plotted against $\theta$ (Figure $4 \mathrm{~d}$ ) and found to demonstrate the same trends as those of the photocurrent densities. It is worthy to note that despite the low areal fraction $(\sim 12.7 \%)$, sparsely arrayed MRs of $4.6 \mu \mathrm{m}$ in height afforded $83.0 \%$ light absorption that is very close to that (86.1\%) of densely arrayed NRs of $4.5 \mu \mathrm{m}$ in height even though the latter has a much higher areal fraction $(\sim 63.0 \%)$. In a same substrate area, the ratios of the numbers of NRs and MRs, their total surface areas, and their photocurrents generated at $V_{\text {bias }}=0.4 \mathrm{~V}$ of these two arrays are $\sim 230: 1$, $\sim 33.4: 1$, and $\sim 4.5: 1$, respectively. Thus, the ratio of photocurrents generated by one NR and one MR is $\sim 1: 50$, which is substantial if considering that the corresponding surface area ratio is $\sim 1: 6.8$. In other words, increasing the packing densities and heights while maintaining quick electrolyte penetration should further enhance the photocurrent densities of sparsely arrayed rods over wide angles of incidence.

\section{CONCLUSIONS}

In short, densely arrayed $\mathrm{ZnO}-\mathrm{NRs}$ and sparsely arrayed $\mathrm{ZnO}$ MRs of systematically varied heights were fabricated in facile and scalable fashions, and their height- and angle-of-incidencedependent PEC performances in water photoelectrolysis were studied via both benchtop measurements and theoretical simulations. For densely arrayed NRs, the electrolyte penetration depth has a limit $(\sim 1.8 \mu \mathrm{m})$, the light absorption only happens at the top regions of NRs up to several hundreds of nanometers, and the reflection is high at all studied angles of incidence $\left(0-60^{\circ}\right)$. In contrast, for sparsely arrayed MRs, the electrolyte penetration is fast and can go at least $\sim 4.6 \mu \mathrm{m}$ or even deeper, the light absorption happens on the entire MR arrays, and the reflection is low at all angles of incidence. These new discoveries should shed new light on the designs and fabrications of arrayed semiconductor rods for both photovoltaic and PEC applications. ${ }^{18}$

\section{ASSOCIATED CONTENT}

\section{S Supporting Information}

PXRD patterns and PL spectra of densely arrayed NRs and sparsely arrayed MRs and their linear sweep voltammagrams under dark and different light illumination, surface areas calculations, and simulated light absorption. SEM images of densely arrayed NRs of $\sim 0.9, \sim 3.6$, and $\sim 4.5 \mu \mathrm{m}$ in height and sparsely arrayed MRs of $\sim 3.6 \mu \mathrm{m}$ in height. This material is available free of charge via the Internet at http://pubs.acs.org.

\section{AUTHOR INFORMATION}

\section{Corresponding Author}

*E-mail: rtian@uark.edu; yangxia@mst.edu.

\section{Funding}

NSF-MRSEC, NSF-EPSCoR, ABI, University of Arkansas, Missouri University of Science and Technology, are acknowledged for their financial support.

\section{Notes}

The authors declare no competing financial interest.

\section{ACKNOWLEDGMENTS}

The project has been partially supported by ABI and NSF through EPSCoR and MRSEC programs. We thank Drs. Kenneth J. Balkus, Alan J. Bard, Kenneth J. Klabunde, Alexandria Navrotsky, Gabor A. Somorjai, Steve L. Suib, and Min Xiao for helpful discussions.

\section{REFERENCES}

(1) Yang, P. D.; Yan, R. X.; Fardy, M. Semiconductor Nanowire: What's Next? Nano Lett. 2010, 10, 1529-1536.

(2) Hochbaum, A. I.; Yang, P. D. Semiconductor Nanowires for Energy Conversion. Chem. Rev. 2010, 110, 527-546. 
(3) Boettcher, S. W.; Spurgeon, J. M.; Putnam, M. C.; Warren, E. L.; Turner-Evans, D. B.; Kelzenberg, M. D.; Maiolo, J. R.; Atwater, H. A.; Lewis, N. S. Energy-Conversion Properties of Vapor-Liquid-SolidGrown Silicon Wire-Array Photocathodes. Science 2010, 327, 185187.

(4) Law, M.; Greene, L. E.; Johnson, J. C.; Saykally, R.; Yang, P. Nanowire Dye-Sensitized Solar Cells. Nat. Mater. 2005, 4, 455-459. (5) Walter, M. G.; Warren, E. L.; McKone, J. R.; Boettcher, S. W.; Mi, Q.; Santori, E. A.; Lewis, N. S. Solar Water Splitting Cell. Chem. Rev. 2010, 110, 6446-6473.

(6) Yu, R.; Ching, K. L.; Lin, Q. F.; Leung, S. F.; Arcrossito, D.; Fan, Z. Y. Strong Light Absorption of Self-Organized 3-D Nanospike Arrays for Photovoltaic Applications. ACS Nano 2011, 5, 9291-9298.

(7) Poxson, D. J.; Kuo, M. L.; Mont, F. W.; Kim, Y. S.; Yan, X.; Welser, R. E.; Sood, A. K.; Cho, J.; Lin, S. Y.; Schubert, E. F. HighPerformance Antireflection Coatings Utilizing Nanoporous Layers. MRS Bull. 2011, 36, 434-438.

(8) Kelzenberg, M. D.; Boettcher, S. W.; Petykiewicz, J. A.; TurnerEvans, D. B.; Putnam, M. C.; Warren, E. L.; Spurgeon, J. M.; Briggs, R. M.; Lewis, N. S.; Atwater, H. A. Enhanced Absorption and Carrier Collection in Si Wire Arrays for Photovoltaic Applications. Nat. Mater. 2010, 9, 239-244.

(9) Greene, L. E.; Law, M.; Tan, D. H.; Montano, M.; Goldberger, J.; Somorjai, G.; Yang, P. D. General Route to Vertical ZnO Nanowire Arrays Using Textured ZnO Seeds. Nano Lett. 2005, 5, 1231-1236.

(10) Xu, S.; Lao, C. S.; Weintraub, B.; Wang, Z. L. DensityControlled Growth of Aligned $\mathrm{ZnO}$ Nanowire Arrays by Seedless Chemical Approach on Smooth Surfaces. J. Mater. Res. 2008, 23, 2072-2077.

(11) Moharam, M. G.; Grann, Eric, B.; Pommet, D. A.; Gaylord, T. K. Formulation for Stable and Efficient Implementation of the Rigorous Coupled-Wave Analysis of Binary Gratings. J. Opt. Soc. Am. A 1995, 12, 1068-1076.

(12) He, Y. R.; Deng, H. X.; Jiao, X. Y.; He, S. L.; Gao, J.; Yang, X. D. Infrared Perfect Absorber Based on Nanowire Metamaterial Cavities. Opt. Lett. 2013, 38, 1179-1181.

(13) Baxter, J. B.; Walker, A. M.; van Ommering, K.; Aydil, E. S. Synthesis and Characterization of $\mathrm{ZnO}$ Nanowires and Their Integration into Dye-Sensitized Solar Cells. Nanotechnology 2006, 17, S304-S312.

(14) Kim, D. H.; Macak, J. M.; Schimidt-Stein, F.; Schmuki, P. Capillary Effects, Wetting Behavior and Photo-Induced Tube Filling of $\mathrm{TiO}_{2}$ Nanotube Layers. Nanotechnology 2008, 19, 305710.

(15) Zhou, Z. J.; Fan, J. Q.; Wang, X.; Zhou, W. H.; Du, Z. L.; Wu, S. X. Effect of Highly Ordered Single-Crystalline $\mathrm{TiO}_{2}$ Nanowire Length on the Photovoltaic Performance of Dye-Sensitized Solar Cells. ACS Appl. Mater. Interfaces 2011, 3, 4349-4353.

(16) Cao, B. Q.; Cai, W. P.; Duan, G. T.; Li, Y.; Zhao, Q.; Yu, D. P. A Template-Free Electrochemical Deposition Route to $\mathrm{ZnO}$ Nanoneedle Arrays and Their Optical and Field Emission Properties. Nanotechnology 2005, 16, 2567-2574.

(17) Linic, S.; Christopher, P.; Ingram, D. B. Plasmonic-Metal Nanostructures for Efficient Conversion of Solar to Chemical Energy. Nat. Mater. 2011, 10, 911-921.

(18) Dasgupta, N. P.; Yang, P. D. Semiconductor Nanowires for Photovoltaic and Photoelectrochemical Energy Conversion. Front. Phys. 2013, DOI: 10.1007/s11467-0305-0. 